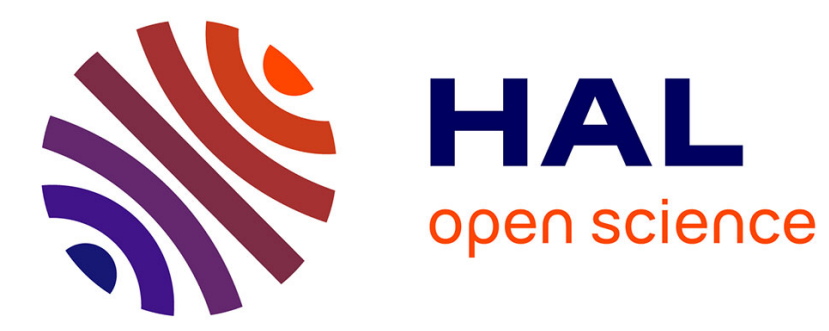

\title{
Latest developments for the Matlab CONTSID toolbox
}

\author{
Hugues Garnier, Marion Gilson, Olivier Cervellin
}

\section{To cite this version:}

Hugues Garnier, Marion Gilson, Olivier Cervellin. Latest developments for the Matlab CONTSID toolbox. 14th IFAC Symposium on System Identification, SYSID'2006, Mar 2006, Newcastle, Australia. pp.714-719. hal-00109102

\section{HAL Id: hal-00109102 \\ https://hal.science/hal-00109102}

Submitted on 23 Oct 2006

HAL is a multi-disciplinary open access archive for the deposit and dissemination of scientific research documents, whether they are published or not. The documents may come from teaching and research institutions in France or abroad, or from public or private research centers.
L'archive ouverte pluridisciplinaire HAL, est destinée au dépôt et à la diffusion de documents scientifiques de niveau recherche, publiés ou non, émanant des établissements d'enseignement et de recherche français ou étrangers, des laboratoires publics ou privés. 


\title{
LATEST DEVELOPMENTS FOR THE MATLAB CONTSID TOOLBOX
}

\author{
Hugues Garnier, Marion Gilson and Olivier Cervellin
}

\author{
Centre de Recherche en Automatique de Nancy, \\ CRAN UMR 7039 CNRS-INPL-UHP \\ Université Henri Poincaré, Nancy 1, BP 239, F-54506 \\ Vandouvre-lès-Nancy Cedex, France, \\ email: firstname.surname@cran.uhp-nancy.fr
}

\begin{abstract}
This paper describes the latest developments for the CONTSID toolbox which includes time-domain identification methods for estimating continuous-time transfer function or state-space models directly from sampled data. The main additions to the new version aim at extending the optimal instrumental variable method to handle wider practical situations in order to enhance the application field of the CONTSID toolbox. The toolbox now includes: (1) a recursive version of the optimal instrumental variable method, (2) a version for multiple input system identification where the denominators of the transfer functions associated with each input are not constrained to be identical, (3) a version for identifying hybrid models of the general Box-Jenkins transfer function form, where a continuous-time plant model with a discrete-time noise model is estimated.
\end{abstract}

Keywords: continuous-time model, discrete-time data, linear system, Matlab toolbox, software tools, system identification.

\section{INTRODUCTION}

This paper ${ }^{1}$ describes the latest developments for the CONtinuous-Time System IDentification (CONTSID) toolbox. This toolbox for use with Matlab was first released in 1999 (Garnier and Mensler, 1999). It has gone through several updates, some of which have been reported at earlier IFAC SYSID Symposia (Garnier and Mensler, 2000; Garnier et al., 2003a). The relevance of direct continuous-time (CT) model identification methods available in the toolbox has been recently illustrated with extensive numerical simulation (Rao and Garnier, 2004). The recent rewriting of the CONTSID toolbox functions as object-

\footnotetext{
1 This report is related to the paper presented at the 14 th IFAC Symposium on System Identification (SYSID'2006), Newcastle (Australia), pp. 714-719, March 2006.
}

oriented to ensure the exchange with the Matlab System Identification toolbox (SITB) has also significantly extended the usability of the toolbox (Garnier et al., 2003a). Amongst the different estimators available for CT SISO system identification (Garnier et al., 2003b), there is one which has proven to be very effective and robust in practical situations. This approach is the Simplified Refined Instrumental Variable for Continuous-time systems (Garnier and Young, 2004), denoted by SRIVC from hereon. It presents the advantage of yielding asymptotically efficient estimates in the presence of white measurement noise. The main additions to the latest version all aim at extending this SRIVC method to enhance its application field. The three main additions concern

- a recursive implementation of the SRIVC method, 
- the development of a SRIVC version for multiple input systems described by multiple transfer functions with different denominators,

- the development of an optimal IV approach for identifying hybrid Box-Jenkins models, where the relationship between the measured input and output is a CT transfer function, while the noise is represented as a discretetime (DT) AR or ARMA process.

This paper is organized as follows. A short overview of the toolbox is given in Section 2 . Section 3 outlines the main steps of the SRIVC method for SISO transfer function models. The recent developments for the toolbox are then presented in Section 4. These latter are illustrated with the help of numerical examples in Section 5 . In Section 6, some of the development plans are briefly discussed.

\section{A SHORT OVERVIEW OF THE CONTSID TOOLBOX}

The CONTSID Matlab toolbox contains timedomain identification methods of $\mathrm{CT}$ parametric models for linear time-invariant SISO and MIMO systems operating in open-loop from regularly or irregularly sampled data. It is designed as an add-on to the System IDentification toolbox (Ljung, 2003) and has a similar setup. Version 4.1 is compatible with Matlab 7.x and operates exclusively in the Matlab command window. This updated version of the CONTSID toolbox is freely available for academic researchers and can be downloaded from:

http://www.cran.uhp-nancy.fr/contsid/

The toolbox contains most of the methods developed over the last thirty years (Garnier et al., 2003b). Parameter estimation techniques can be divided into the following two families:

(1) transfer function model estimation schemes to identify SISO or MISO systems,

(2) state-space model estimation schemes to identify MIMO systems.

The main demonstration program called idcdemo.m provides several examples illustrating the use of the CONTSID toolbox approaches.

\section{SRIVC FOR SISO TF MODEL}

Consider a linear, single-input, single-output, CT system $^{2}$ whose input $u(t)$ and output $y(t)$ are related by a constant coefficient differential equation

\footnotetext{
2 A time-delay on the system input is not considered for simplicity here but is easy to accommodate.
}

$$
\begin{aligned}
& x^{(n)}(t)+a_{1} x^{(n-1)}(t)+\ldots+a_{n} x^{(0)}(t)= \\
& b_{0} u^{(m)}(t)+b_{1} u^{(m-1)}(t)+\ldots+b_{m} u^{(0)}(t)
\end{aligned}
$$

where $x^{(i)}(t)$ denotes the $i$ th time-derivative of the continuous-time signal $x(t)$. Equation (1) can also be written in the transfer function (TF) form:

$$
x(t)=\frac{B(s)}{A(s)} u(t)
$$

with

$$
\begin{aligned}
& B(s)=b_{0} s^{m}+b_{1} s^{m-1}+\cdots+b_{m}, \\
& A(s)=s^{n}+a_{1} s^{n-1}+\cdots+a_{n},
\end{aligned}
$$

where $s$ is the differential operator, i.e. $s^{p} x(t)=\frac{d^{p} x(t)}{d t^{p}}$. It is assumed that the input signal $\left\{u(t), t_{1}<t<t_{N}\right\}$ is applied to the system and that the output $x(t)$ is sampled at discrete times $t_{1}, \cdots, t_{N}$, not necessarily uniformly spaced.

In order to obtain high quality statistical estimation results, it is vital to consider the inevitable errors that will affect the measured output signal. In the case of uniformly sampled data, the measured output $y_{k}=y\left(t_{k}\right)$, where $t_{k}=k T_{s}$, is assumed to be corrupted by an additive measurement noise $\xi\left(t_{k}\right)$

$$
y\left(t_{k}\right)=x\left(t_{k}\right)+\xi\left(t_{k}\right) .
$$

where $x\left(t_{k}\right)$ is the hypothetical noise-free, deterministic output of the system. The identification problem aims at estimating the parameters of the differential equation model (2) from $N$ sampled measurements of the input and output $Z^{N}=$ $\left\{u\left(t_{k}\right) ; y\left(t_{k}\right)\right\}_{k=1}^{N}$.

One particularly successful stochastic identification method is the iterative Simplified Refined Instrumental Variable method for Continuous-time model Identification (SRIVC: see Young and Jakeman (1980), Young (2002)) where, for simplicity, the noise is assumed to be discrete-time, white noise process so that no explicit noise modelling is necessary, except in relation to the estimation of the variance of the white noise process. This approach involves a method of adaptive prefiltering based on a quasi-optimal ${ }^{3}$ statistical solution to the problem in this white noise case.

The SRIVC algorithm is based on the assumption that the additive noise $\xi\left(t_{k}\right)$ in (3) is simply a zero mean, normally distributed, white noise process $e\left(t_{k}\right)$ : i.e. $e\left(t_{k}\right)=N\left(0, \sigma^{2}\right)$. In this situation, following the usual Prediction Error Minimization (PEM) approach (which is Maximum Likelihood (ML) estimation in the present situation because

\footnotetext{
3 The method is quasi-optimal because true optimality would require optimal interpolation of the input signal $u(t)$ over the sampling interval, whereas only simple interpolation is used in the SRIVC implementation. However, this normally produces very good, near optimal estimation results.
} 
of the Gaussian assumption on $e\left(t_{k}\right)$ ), a suitable error function $\varepsilon(t)$ in continuous-time is given by the output error $(\mathrm{OE})$,

$$
\varepsilon(t)=y(t)-\frac{B(s)}{A(s)} u(t),
$$

which can be written as,

$$
\varepsilon(t)=\frac{1}{A(s)}[A(s) y(t)-B(s) u(t)] .
$$

Minimization of a least squares criterion function in $\varepsilon\left(t_{k}\right)$, measured at the sampling instants, provides the basis for stochastic estimation. However, since the polynomial operators commute in this linear case, the filter $F(s)=1 / A(s)$ can be taken inside the square brackets to yield:

$$
\varepsilon(t)=A(s) y_{f}(t)-B(s) u_{f}(t)
$$

or,

$$
\begin{aligned}
\varepsilon(t) & =y_{f}^{(n)}(t)+a_{1} y_{f}^{(n-1)}(t)+\ldots+a_{n} y_{f}^{(0)}(t) \\
& -b_{0} u_{f}^{(m)}(t)-\ldots-b_{m} u_{f}^{(0)}(t)
\end{aligned}
$$

where

$$
\begin{cases}y_{f}^{(i)}(t)=f_{i}(t) * y(t), & i=0, \ldots, n \\ u_{f}^{(i)}(t)=f_{i}(t) * u(t), & i=0, \ldots, m,\end{cases}
$$

The * symbol denotes the convolution operator and the set of filters takes the form

$$
f_{i}(t)=\mathcal{L}^{-1}\left(\frac{s^{i}}{A(s)}\right)
$$

where $\mathcal{L}^{-1}$ is the inverse Laplace transform. So that, at the $k^{t h}$ sampling instant $t=t_{k}$, the associated estimation model can be written in the form:

$$
y_{f}^{(n)}\left(t_{k}\right)=\phi_{f}^{T}\left(t_{k}\right) \theta+\varepsilon\left(t_{k}\right)
$$

where $\phi_{f}^{T}\left(t_{k}\right)$ and $\theta$ are defined as

$$
\begin{gathered}
\phi_{f}^{T}\left(t_{k}\right)=\left[\begin{array}{lll}
-y_{f}^{(n-1)}\left(t_{k}\right) & \cdots-y_{f}^{(0)}\left(t_{k}\right) \\
u_{f}^{(m)}\left(t_{k}\right) & \cdots & u_{f}^{(0)}\left(t_{k}\right)
\end{array}\right] \\
\theta=\left[\begin{array}{llllll}
a_{1} & \ldots & a_{n} & b_{0} & \ldots & b_{m}
\end{array}\right]^{T} .
\end{gathered}
$$

Thus, provided we assume that $A(s)$ is known a priori, the estimation model (9) forms a basis for the definition of a likelihood function and ML estimation.

There are two problems with this formulation. The most obvious one is, of course, that the $A(s)$ polynomial is not known a priori. The less obvious one is that, in practical applications, we cannot assume that the noise $\xi\left(t_{k}\right)$ will always be white: in most applications it will be coloured. Both of these problems can be solved by employing a similar approach to that used first in the RIV algorithm for DT system identification and estimation. Here, a 'relaxation' optimization procedure is devised that iteratively adjusts the unknown polynomials in the TF model (2), as well as the estimate of the instrumental variable, until they converge.
The instrumental variable at each iteration of the algorithm is generated by the following auxiliary model

$$
\hat{x}(t)=\frac{B\left(s, \hat{\theta}_{N}^{I V}\right)}{A\left(s, \hat{\theta}_{N}^{I V}\right)} u(t) .
$$

where $\hat{\theta}_{N}^{I V}$ is the estimated parameter vector obtained at the previous iteration of the algorithm. The main steps in this algorithm are given in Young (2002).

\section{LATEST DEVELOPMENTS}

\subsection{Recursive implementation of the SRIVC method}

In many situations, we need to estimate the model at the same time as the data is collected during the measurement. The model is then "updated" at each time instant some new data becomes available. The updating is performed by a recursive algorithm for system identification. The SRIVC method outlined above can easily be applied as on-line algorithms in a recursive form and is now available in the CONTSID toolbox.

\subsection{SRIVC for MISO transfer function models}

In DT model estimation, the approaches dedicated to multiple transfer function model identification combine either extensions of linear regression techniques like pseudo-linear, multi-linear regression, instrumental variable, or non linear optimization techniques (Ljung, 1999). For the CT case, to our knowledge, the only procedure developed to handle the MISO identification problem was based on non linear optimization techniques which minimize the output error. However, these techniques may critically rely on a good initial parameter set to converge to the global minimum of the cost function. The linear regression-based algorithms offer an interesting solution to overcome this drawback. However, the parameter estimation procedures for MISO systems have usually been developed by a straightforward extension of procedures devoted to SISO systems, which only allows transfer function estimation with a common denominator. Since this case is not very realistic in many practical applications, a new method to estimate MISO systems described by multiple CT transfer functions with different denominators has been recently developed (Huselstein et al., 2004). The main idea is recalled here.

Consider a MISO CT linear time-invariant causal system that can be described by

$$
\left\{\begin{array}{l}
x_{i}\left(t_{k}\right)=G_{i}(s) u_{i}\left(t_{k}\right), \\
x\left(t_{k}\right)=\sum_{i=1}^{n_{u}} x_{i}\left(t_{k}\right), \\
y\left(t_{k}\right)=x\left(t_{k}\right)+\xi\left(t_{k}\right),
\end{array}\right.
$$


where $G_{i}(s)$ is the $i$ th transfer function given by

$$
\begin{aligned}
G_{i}(s) & =\frac{B_{i}(s)}{F_{i}(s)}=\frac{b_{i, 0}+b_{i, 1} s+\cdots+b_{i, m_{i}} s^{m_{i}}}{f_{i, 0}+f_{i, 1} s+\cdots+f_{i, n_{i}} s^{n_{i}}}, \\
f_{i, n_{i}} & =1, \quad n_{i} \geq m_{i}, \quad i=1, \ldots, n_{u},
\end{aligned}
$$

and $\theta_{i}=\left[\begin{array}{llllll}b_{i, m_{i}} & \ldots & b_{i, 0} & f_{i, n_{i}-1} & \ldots & f_{i, 0}\end{array}\right]^{T} \in \mathbb{R}^{n_{s_{i}}}$, with $n_{s_{i}}=n_{i}+m_{i}+1$, where $n_{i}$ and $m_{i}$ denote the denominator and numerator orders of $G_{i}(s)$ respectively. Therefore, the sought parameter vector is

$$
\theta=\left[\begin{array}{lll}
\theta_{1}^{T} & \ldots & \theta_{n_{u}}^{T}
\end{array}\right]^{T} \in \mathbb{R}^{n_{p} \times 1},
$$

with $n_{p}=\sum_{i=1}^{n_{u}} n_{p_{i}}$.

The proposed method ${ }^{4}$ aims at identifying MISO model with different denominators for each input (13), which is more realistic than assuming an identical denominator for all transfer functions. However, the model is no longer linear in the parameters and the proposed MISO version of SRIVC lies, therefore, in the domain of multilinear regression. The MISO model (13) can be converted into $n_{u}$ SISO models as follows

$$
\begin{aligned}
v\left(t_{k}, \theta\right) & =\xi_{i, f}\left(t_{k}, \theta\right)-x_{i, f}\left(t_{k}, \theta_{i}\right) \\
\xi_{i, f}\left(t_{k}, \theta\right) & =y_{f}\left(t_{k}\right)-\sum_{j=1, j \neq i}^{n_{u}} x_{j, f}\left(t_{k}, \theta_{j}\right)
\end{aligned}
$$

The parameter vector $\theta$ is partitioned into classes $\theta_{1}, \ldots, \theta_{n_{u}}$ such that the error is affine with respect to the parameters of any of these classes when the parameters of all others are fixed. It is then possible to search for $\hat{\theta}$ by applying successively the SISO version of the SRIVC to estimate the parameters of each class in turn, with a cyclic exploration of all classes. This is achieved by following the same type of 'relaxation' procedure described in Section 3.

A key point to be solved in the identification procedure concerns the model order selection. The method available for SISO systems based on the properties of the instrumental product matrix (see e.g. (Young, 2002)) has been extended to the case of MISO systems for identifying the model structure prior to parameter estimation.

\subsection{RIVC method for identifying hybrid Box-Jenkins models}

If the noise $\xi\left(t_{k}\right)$ in the observation equation (3) is coloured and described by an $\operatorname{ARMA}(p, q)$ model, then the SRIVC method has no claim to optimality in statistical terms. However, because it inherently includes the IV mechanism, the estimates are consistent. Moreover, experience has

\footnotetext{
4 The approach is also available in the CAPTAIN Toolbox for Matlab: http://www.es.lancs.ac.uk/cres/captain/
}

shown that the algorithm is very robust in practical application and normally yields estimates with reasonable statistical efficiency (i.e. low but not minimum variance). Nevertheless, it is worthwhile extending the algorithm to the coloured noise case by employing the hybrid approach adumbrated above, where the noise modelling, as well as the noise-derived parts of the prefiltering, are carried out in discrete-time terms.

In general, following the normal approach to the estimation of stochastic TF models, it makes sense to assume that the discrete-time noise process $\xi\left(t_{k}\right)$ in (3) has rational spectral density and so can be described by an $\operatorname{ARMA}(p, q)$ process, i.e.,

$$
\xi\left(t_{k}\right)=\frac{C\left(z^{-1}\right)}{D\left(z^{-1}\right)} e\left(t_{k}\right) \quad e\left(t_{k}\right)=N\left(0, \sigma^{2}\right)
$$

where,

$$
\begin{aligned}
& C\left(z^{-1}\right)=1+c_{1} z^{-1}+\ldots+c_{p} z^{-p} \\
& D\left(z^{-1}\right)=1+d_{1} z^{-1}+\ldots+d_{q} z^{-q}
\end{aligned}
$$

are, respectively, the Moving Average (MA) and Autoregressive (AR) polynomials in the backward shift operator, denoted here by $z^{-r}$; i.e. $z^{-r} y_{k}=$ $y_{k-r}$.

Following the approach used in the SRIVC case, we need to consider a hybrid prefiltering operation involving both continuous and discrete-time prefilters. In particular, since discrete-time noise (18) is only associated with the definition of the observation equation (3) and we are dealing with a linear model, the prefiltering can be considered in these two stages: first, the continuoustime prefiltering already described in the SRIVC algorithm above; and second, discrete-time prefiltering based on the inverse of the $\operatorname{ARMA}(p, q)$ noise model.

In order to explain this hybrid prefiltering, consider the discrete-time sampled value $\varepsilon\left(t_{k}\right)$ of $\varepsilon(t)$ in (4). Introducing the $\operatorname{ARMA}(p, q)$ noise process (18), the RIVC error function $\epsilon\left(t_{k}\right)$, now defined in discrete-time, can be written as,

$$
\epsilon\left(t_{k}\right)=\frac{D\left(z^{-1}\right)}{C\left(z^{-1}\right)} \varepsilon\left(t_{k}\right)
$$

where $\varepsilon\left(t_{k}\right)$ is given by (5) and the DT prefilter will be recognized as the inverse of the $\operatorname{ARMA}(p, q)$ noise model. With this definition, the estimation model takes a similar form to (9), i.e.:

$$
y_{f}^{(n)}\left(t_{k}\right)=\phi_{f}^{T}\left(t_{k}\right) \theta+\epsilon\left(t_{k}\right)
$$

but the prefilters required in the definition of the estimation model are obviously more complex, requiring the practical implementation of the above hybrid filtering operations. The main steps of the full RIVC algorithm are outlined in Young et al. (2006). 


\section{EXAMPLES OF THE NEW FEATURES}

The new developments are illustrated with the help of numerical examples in this section.

\subsection{Recursive identification}

The following example shows the recursive SRIVC algorithm being used to identify a CT second order SISO system without delay described by

$$
\begin{aligned}
x(t) & =\frac{4}{s^{2}+3 s+2} u(t), \\
y\left(t_{k}\right) & =x\left(t_{k}\right)+\xi\left(t_{k}\right) .
\end{aligned}
$$

The input signal stored in $u$ is chosen as a PRBS of maximum length. 2040 data points are used for the identification. The sampling period is set to $10 \mathrm{~ms} . \xi\left(t_{k}\right)$ is chosen here as a zero-mean white Gaussian noise which variance is adjusted to get a signal-to-noise ratio of $20 \mathrm{~dB}$. The simulated output is stored in $\mathrm{y}$.

Let us now recursively identify a CT model from the sampled data $\mathrm{z}=\mathrm{iddata}\left(\mathrm{y}, \mathrm{u}, T_{s}\right)$, by the recursive version of optimal IV algorithm rsrivc. The extra information needed are

- the number of parameters for the TF numerator and denominator and number of sample for the delay of the model stored in the variable $n n=\left[\begin{array}{lll}n b & n f & n k\end{array}\right]=\left[\begin{array}{lll}1 & 2 & 0\end{array}\right]$,

- lambda which is the cut-off frequency (in $\mathrm{rad} / \mathrm{s}$ ) of the filter used to get an initial parameter estimate ( lambda $=2$ ).

The routine can now be used as follows:

$[\mathrm{m}, \mathrm{thm}]=\operatorname{rsrivc}(\mathrm{z}, \mathrm{nn}$, lambda $)$;

The final estimated model is stored in $\mathrm{m}$ and can be displayed with: present (m)

which leads to:

CT IDPOLY model: $y(t)=[B(s) / F(s)] u(t)$

$\mathrm{B}(\mathrm{s})=3.990$

$F(s)=s^{2}+3.031 s+2.004$

Loss function 0.00089 and FPE 0.00089

Estimated using RSRIVC

The second output argument thm is a matrix that contains the estimated model parameters at the different samples. The estimated parameter estimates can then be plotted as a function of time, as displayed in Figure 1.

\subsection{MISO transfer function identification}

The following example shows the SRIVC algorithm being used to identify a 2 inputs 1 output system described by

$$
\begin{aligned}
x(t) & =\frac{4 e^{-3 T_{s} s}}{s^{2}+2 s+4} u_{1}(t)+\frac{e^{-5 T_{s} s}}{s^{2}+s+1} u_{2}(t), \\
y\left(t_{k}\right) & =x\left(t_{k}\right)+\xi\left(t_{k}\right) .
\end{aligned}
$$

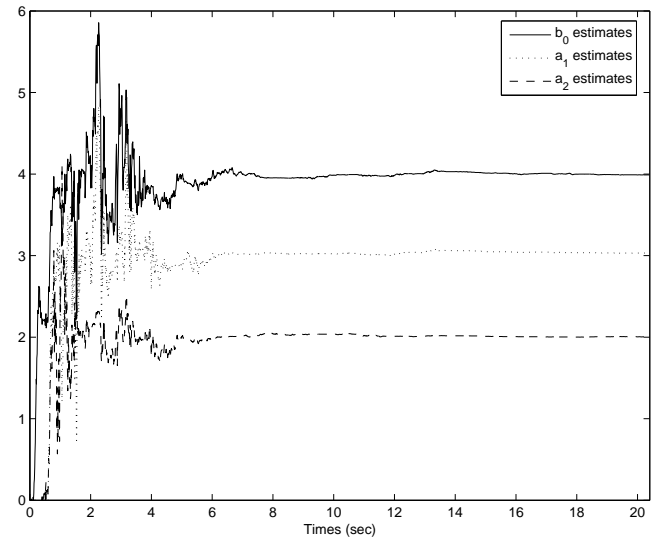

Fig. 1. RSRIVC estimates as a function of time

The two inputs stored in [u1 u2] are chosen as uncorrelated PRBS of maximum length. The sampling period is set to $10 \mathrm{~ms}$. $\xi\left(t_{k}\right)$ is chosen here as a zero-mean white Gaussian noise which variance is adjusted to get a signal-to-noise ratio of $10 \mathrm{~dB}$. The noisy output is stored in $\mathrm{y}$.

Let us now identify a CT model from the sampled data

$\mathrm{z}=$ iddata $\left(\mathrm{y}\right.$, [u1 u2] , $T_{s}$ ), by the MISO version of optimal IV algorithm srivc. The extra information needed in this case are

- the number of parameters for the TF numerators and denominators and numbers of sample for the delays of the model stored in $\mathrm{nn}=\left[\begin{array}{llllll}n b 1 & \mathrm{nb} 2 & \mathrm{nf} 1 & \mathrm{nf} 2 & \mathrm{nk} 1 & \mathrm{nk} 2\end{array}\right]=\left[\begin{array}{llllll}1 & 1 & 2 & 2 & 3 & 5\end{array}\right]$

- lambda which is the cut-off frequency (in $\mathrm{rad} / \mathrm{s}$ ) of the filter used to get an initial parameter estimate ( $\mathrm{lambda}=3$ ).

The routine can now be used as follows:

$\mathrm{m}=\operatorname{srivc}(\mathrm{z}, \mathrm{nn}, \mathrm{lambda})$;

The final estimated model is stored in $\mathrm{m}$ and can be displayed with:

present (m)

which leads to:

CT IDPOLY model: $\mathrm{y}(\mathrm{t})=[\mathrm{B}(\mathrm{s}) / \mathrm{F}(\mathrm{s})] \mathrm{u}(\mathrm{t})$

$\mathrm{B} 1(\mathrm{~s})=4.08(+-0.0786)$

$\mathrm{B} 2(\mathrm{~s})=0.9869(+-0.0307)$

$\mathrm{F} 1(\mathrm{~s})=\mathrm{s}^{2}+1.952(+-0.0599) \mathrm{s}+3.906(+-0.0750)$

$\mathrm{F} 2(\mathrm{~s})=\mathrm{s}^{2}+1.002(+-0.0552) \mathrm{s}+1.01(+-0.0319)$

Input delays (listed by channel): 0.030 .05

Estimated using SRIVC

Loss function 0.00664518 and FPE 0.00670851

The figures in parentheses are the estimated standard deviation of the associated parameter estimates. More illustration of the use of the MISO SRIVC version can be obtained by executing idcdemo3.

\subsection{Hybrid Box-Jenkins model identification}

This last example shows the RIVC algorithm being used to identify the hybrid Box-Jenkins 
model. The system and the simulation conditions are identical to the ones used in Section 5.1. $\xi\left(t_{k}\right)$ is now an $\operatorname{AR}(2)$ noise model given by

$$
\xi\left(t_{k}\right)=\frac{1}{1-q^{-1}+0.2 q^{-2}} e\left(t_{k}\right)
$$

where $e\left(t_{k}\right)$ is chosen here as a zero-mean white Gaussian noise. The variance of $\xi\left(t_{k}\right)$ is adjusted to get a signal-to-noise ratio of $10 \mathrm{~dB}$. The full hybrid version of the optimal IV algorithm rivc can then be used by specifying the extra information needed in this case

- the number of parameters for the TF numerator $\mathrm{nb}$ and denominator $\mathrm{nf}$ and number of sample for the delay $n k$ but also the number of parameters for the AR model nd stored in $\mathrm{nn}=\left[\begin{array}{lll}n b & n f & n k \\ n d\end{array}\right]=\left[\begin{array}{llll}1 & 2 & 0 & 2\end{array}\right]$

- lambda, the cut-off frequency of an all-pole filter used to get an initial parameter estimate $(\text { lambda }=2)^{5}$.

The routine can then be used as follows:

$[\mathrm{m}, \mathrm{D}]=\operatorname{rivc}(\mathrm{z}, \mathrm{nn}, \mathrm{lambda})$;

The estimated model stored in $\mathrm{m}$ can be displayed CT IDPOLY model: $y(t)=[B(s) / F(s)] u(t)$

$B(s)=4.292$

$F(s)=s^{2}+3.077 s+2.198$

Input delays (listed by channel): 0

Estimated using RIVC

Loss function 0.00259509 and FPE 0.00260274

The second output argument $\mathrm{D}$ is a vector that contains the estimated AR model parameter $D\left(q^{-1}\right)=1-1.0091 q^{-1}+0.2178 q^{-2}$

\section{DEVELOPMENT PLANS}

The new developments outlined in this paper significantly extend the practical application field of the CONTSID toolbox. We plan in the near future to concentrate on the following developments:

- writing of a user's guide,

- implementation of several instrumental variable based methods for identifying continuous-time models of systems operating in closed-loop (Gilson et al., 2004),

- implementation of a graphical user interface. We are working on the development of an easy-to-use interface which allows the user to perform identification, data and model analysis, as well as model validation by "click and mouse" operations.

\footnotetext{
5 The initiation of the rivc algorithm involves the selection of a suitable prefilter. The option chosen here is to specify a suitable cut-off frequency of the filter. However the default option uses the DT RIV algorithm to estimate first a DT model which is then transformed to $\mathrm{CT}$ form and provides automatically the required filter.
}

\section{REFERENCES}

Garnier, H. and M. Mensler (1999). CONTSID: a continuous-time system identification toolbox for Matlab. In: 5th European Control Conference (ECC'99). Karlsruhe (Germany).

Garnier, H. and M. Mensler (2000). The CONTSID toolbox: a Matlab toolbox for CONtinuous-Time System IDentification. In: 12th IFAC Symposium on System Identification (SYSID'2000). Santa Barbara (USA).

Garnier, H. and P.C. Young (2004). Time-domain approaches for continuous-time model identification from sampled data. In: Invited tutorial paper for the American Control Conference (ACC'2004). Boston, MA (USA).

Garnier, H., M. Gilson and E. Huselsein (2003a). Developments for the Matlab CONTSID toolbox. In: 13th IFAC Symposium on System Identification (SYSID'2003). Rotterdam (The Netherlands). pp. 1007-1012.

Garnier, H., M. Mensler and A. Richard (2003b). Continuous-time model identification from sampled data. Implementation issues and performance evaluation. International Journal of Control 76(13), 1337-1357.

Gilson, M., H. Garnier and P. Van den Hof (2004). Instrumental variable methods for continuous-time model identification in closed-loop. In: American Control Conference (ACC'2004). Boston, MA (USA).

Huselstein, E., M. Gilson, H. Garnier and A. Richard (2004). Instrumental variable algorithms for multiple input systems described by multiple continuous-time TF models. In: Asian Control Conference. Melbourne (Australia).

Ljung, L. (1999). System identification. Theory for the user. 2nd ed.. Prentice Hall. Upper Saddle River.

Ljung, L. (2003). Version 6 of the system identifcation toolbox. In: 13th IFAC Symposium on System Identification (SYSID'2003). Rotterdam (The Netherlands). pp. 989-994.

Rao, G.P. and H. Garnier (2004). Identification of continuous-time systems: direct or indirect?. Systems Science 30(3), 25-50.

Young, P.C. (2002). Optimal IV identification and estimation of continuous-time TF models. In: 15th Triennial IFAC World Congress on Automatic Control. Barcelona (Spain).

Young, P.C. and A.J. Jakeman (1980). Refined instrumental variable methods of time-series analysis: Part III, extensions. International Journal of Control 31, 741-764.

Young, P.C., H. Garnier and M. Gilson (2006). An optimal instrumental variable approach for identifying hybrid continuous-time BoxJenkins model. In: Proceedings of the 14th IFAC Symposium on System Identification (SYSID'2006). Newcastle (Australia). 